Luis A. Florit · Wolfgang Ziller

\title{
On the topology of positively curved Bazaikin spaces
}

\author{
Received May 24, 2007
}

\begin{abstract}
We explore some aspects of the topology of the family of 13-dimensional Bazaikin spaces. Using the computation of their homology rings, Pontryagin classes and linking forms, we show that there is only one Bazaikin space that is homotopy equivalent to a homogeneous space, i.e., the Berger space. Moreover, it is easily shown that there are only finitely many Bazaikin spaces in each homeomorphism type and that there are only finitely many positively curved ones for a given cohomology ring. In fact, supported by computational experiments, it is conjectured that all positively curved Bazaikin spaces are homeomorphically, or at least diffeomorphically, distinct.
\end{abstract}

Keywords. Positive curvature, Bazaikin spaces

Manifolds with positive sectional curvature have been of interest since the beginning of global Riemannian geometry, as illustrated by the theorem of Bonnet-Myers, the Berger-Klingenberg sphere theorem, and the Synge theorem. Surprisingly, for compact simply connected manifolds, no obstructions are known to distinguish between manifolds (that admit a metric) with non-negative curvature and those of positive curvature. On the other hand, among known examples, the class of non-negatively curved manifolds is much larger. They include, among others, any homogeneous space and more generally the so called biquotients, i.e. quotients of a compact Lie group $G$ by a subgroup of $G \times G$ acting on $G$ from the left and right simultaneously. A biinvariant metric on $G$ induces a non-negatively curved metric on the quotient.

The difficulty of finding new examples of manifolds with positive curvature may be illustrated by the fact that all known examples are constructed in this way, allowing more generally left invariant metrics on $G$. But only very few manifolds of this type have positive curvature and, apart from the rank one symmetric spaces, examples exist only in low dimensions. They include certain homogeneous spaces in dimensions 6, 7, 12, 13 and 24 due to Berger [Be], Wallach [Wa], and Aloff-Wallach [AW], and biquotients in dimensions 6, 7 and 13 due to Eschenburg [E1], [E2] and Bazaikin [Ba].

L. A. Florit: IMPA, Est. Dona Castorina 110, 22460-320, Rio de Janeiro, Brazil; e-mail: luis@impa.br

W. Ziller: Department of Mathematics, University of Pennsylvania, Philadelphia, PA 19104, USA; e-mail: wziller@math.upenn.edu

Mathematics Subject Classification (2000): 53C20 
Aside from four isolated manifolds, this list divides into two infinite families. The first in dimension 7 consists of the Eschenburg biquotients

$$
\mathcal{E}_{k, l}=\operatorname{diag}\left(z^{k_{1}}, z^{k_{2}}, z^{k_{3}}\right) \backslash \mathrm{SU}(3) / \operatorname{diag}\left(z^{l_{1}}, z^{l_{2}}, z^{l_{3}}\right)^{-1},
$$

with $\sum k_{i}=\sum l_{i}$, which include the infinite subfamily of homogeneous Aloff-Wallach spaces $\mathcal{W}=\operatorname{SU}(3) / \operatorname{diag}\left(z^{l_{1}}, z^{l_{2}}, z^{l_{3}}\right), \sum l_{i}=0$. The second one exists in dimension 13 and consists of the Bazaikin biquotients (as described in [EKS])

$$
\mathcal{B}_{q}=\operatorname{diag}\left(z^{q_{0}}, z^{q_{1}}, z^{q_{2}}, z^{q_{3}}, z^{q_{4}}, z^{q_{5}}\right) \backslash \mathrm{SU}(6) / \mathrm{Sp}(3),
$$

where $q=\left(q_{0}, \ldots, q_{5}\right)$ are odd integers with $\sum q_{i}=0$. When the restrictions on $q$ that are necessary for this biquotient to be a manifold are satisfied, we call $\mathcal{B}_{q}$ a Bazaikin space. If, in addition, the condition for a certain metric on $\mathcal{B}_{q}$ to have positive sectional curvature holds, we call $\mathcal{B}_{q}$ a positively curved Bazaikin space; see Section 1.

Topological properties of the Eschenburg spaces $\mathcal{E}$ have been studied extensively. A partial classification of their homeomorphism and diffeomorphism types has been obtained in [KS] and [K1], [K2]. Using these, it was shown that occasionally positively curved homogeneous Aloff-Wallach spaces ([KS] $)$, and more frequently positively curved Eschenburg biquotients ([CEZ]), may be homeomorphic to each other, but not diffeomorphic. Furthermore, Eschenburg spaces are frequently homotopy equivalent and occasionally homeomorphic and even diffeomorphic to homogeneous Aloff-Wallach spaces without being Aloff-Wallach spaces by definition; cf. [CEZ]. See also [AMP1], [AMP2], and $[\mathrm{Sh}]$.

In this paper we study topological properties of the Bazaikin spaces and we will see that their behaviour is quite different from the Eschenburg spaces. A further difference is that there is only one Bazaikin space which is homogeneous by definition, the Berger space $\mathrm{SU}(5) / \mathrm{Sp}(2) \mathrm{S}^{1}$ (see $[\overline{\mathrm{Be}}]$ ), which corresponds to $q=(-5,1,1,1,1,1)$ (up to order). On the other hand, there exists a large class of homogeneous spaces in dimension 13. However, we will show:

Theorem A. A Bazaikin space $\mathcal{B}_{q}$ with $q \neq(-5,1,1,1,1,1)$ is not homeomorphic to a homogeneous space. If, in addition, $\mathcal{B}_{q}$ is positively curved, then it is not even homotopy equivalent to a homogeneous space.

We point out that there are positively curved Bazaikin spaces which have the same cohomology ring as certain homogeneous spaces (see Section 3), and there are Bazaikin spaces which match all the homotopy invariants of the Berger space computed in Theorem $B$.

In contrast to Theorem $\mathrm{A}$, there are positively curved Eschenburg spaces that are homotopically equivalent but not homeomorphic to Aloff-Wallach spaces ([Sh]$])$.

The proof involves, among other things, the computation of certain topological invariants. So far no classification of the Bazaikin spaces up to homeomorphism or diffeomorphism has been obtained. In fact, only the cohomology ring was known ([Ba] $)$. The property of the cohomology ring that can distinguish Bazaikin spaces from each other is the order $s$ of the finite torsion groups $H^{6}\left(\mathcal{B}_{q}, \mathbb{Z}\right)=H^{8}\left(\mathcal{B}_{q}, \mathbb{Z}\right)=\mathbb{Z}_{s}$. Further invariants are given by the Pontryagin classes $p_{1} \in H^{4}\left(\mathcal{B}_{q}, \mathbb{Z}\right)=\mathbb{Z}$ and $p_{2} \in H^{8}\left(\mathcal{B}_{q}, \mathbb{Z}\right)=\mathbb{Z}_{s}$, and the linking form $l k \in \mathbb{Z}_{s}$. 
Theorem B. For a Bazaikin space $\mathcal{B}_{q}$ the order $s$ is odd and

$$
\begin{gathered}
8 s=\left|\sigma_{3}\right|, \quad l k= \pm 32 \sigma_{5}^{-1} \in \mathbb{Z}_{s}, \\
p_{1}=-\sigma_{2}=\frac{1}{2}\|q\|^{2}, \quad 8 p_{2}=3 p_{1}^{2}-\sigma_{4} \in \mathbb{Z}_{s},
\end{gathered}
$$

where $\sigma_{i}=\sigma_{i}(q)$ stands for the elementary symmetric polynomial of degree $i$ in $q$.

Recall that $s$ and $l k$, as well as $p_{1} \bmod 24$, are homotopy invariants, while rational Pontryagin classes are homeomorphism invariants. For a Bazaikin space, $p_{1}$ is hence a homeomorphism invariant while $p_{2}$ is a diffeomorphism invariant.

Corollary $\mathbf{C}$. The following finiteness results hold:

(a) There are only finitely many positively curved Bazaikin spaces for a given cohomology ring.

(b) There are only finitely many Bazaikin spaces in each homeomorphism type.

Part (a) is also true for Eschenburg spaces ([CEZ]) and should be viewed in the context of the Klingenberg-Sakai conjecture. It states that there are only finitely many positively curved manifolds in a given homotopy type and raises the question whether this might be true even for a given cohomology ring.

It is not difficult to find Bazaikin spaces that match all four invariants in Theorem $B$, especially for small values of $s$. However, by means of a computer program, we observed that:

$$
\begin{aligned}
& \text { All positively curved Bazaikin spaces with } s \leq 10^{9}, \\
& \text { altogether } 2130601485 \text { manifolds, are homeomorphically distinct. }
\end{aligned}
$$

This strongly suggests that indeed all positively curved Bazaikin spaces are homeomorphically, or at least diffeomorphically, distinct.

In Section 1 we discuss some preliminaries, in Section 2 we compute the homotopy invariants, while in Section 3 we compute the Pontryagin classes and present the proof of Theorem A We end the paper with some computer experiment results in Section 4.

\section{Preliminaries}

A biquotient can be defined in several ways. First, consider two subgroups of $G$ defined by monomorphisms $f_{1}: H \rightarrow G$ and $f_{2}: K \rightarrow G$. The group $H \times K$ acts on $G$ via $(h, k) \cdot g=f_{1}(h) g f_{2}(k)^{-1}$. If this action is free, the quotient $H \backslash G / K$ is called a biquotient. In the literature one often finds the seemingly more general definition where $H \times K$ is allowed to be replaced by a subgroup $L$ of $G \times G$. This can be reduced to the above case by rewriting the biquotient as $\triangle G \backslash G \times G / L$. The action is free iff $(h, k)=(e, e)$ whenever $h \in H$ and $k \in K$ are conjugate to each other. This can clearly be further reduced to the case where we assume that $h$ and $k$ lie in a maximal torus.

In the following we also allow the action of $H \times K$ to have a finite ineffective kernel $\Gamma$ (necessarily embedded diagonally in $H \times K)$ and that $(H \times K) / \Gamma$ acts freely on $G$. This again is not more general since it can be reduced to the above by replacing $G$ with $G / \Gamma$. But the advantage is that the topological computations become simpler when $G$ is simply connected. 
Following $[\overline{E K S}]$, we define a Bazaikin space as

$$
\mathcal{B}_{q}=\operatorname{diag}\left(z^{q_{0}}, z^{q_{1}}, z^{q_{2}}, z^{q_{3}}, z^{q_{4}}, z^{q_{5}}\right) \backslash \mathrm{SU}(6) / \mathrm{Sp}(3),
$$

where $z \in \mathrm{S}^{1}=\{w \in \mathbb{C}:|w|=1\}$ and $q=\left(q_{0}, \ldots, q_{5}\right)$ is an ordered set of integers with $\sum q_{i}=0$. We choose the embedding $\mathrm{Sp}(3) \subset \mathrm{SU}(6)$ such that a maximal torus of $\mathrm{Sp}(3)$ is given by $\operatorname{diag}(u, \bar{u}, v, \bar{v}, w, \bar{w}) \subset \mathrm{SU}(6)$. This biquotient action of $\mathrm{S}^{1} \times \mathrm{Sp}(3)$ on $\mathrm{SU}(6)$ has ineffective kernel $\Gamma=\mathbb{Z}_{2}=\langle(-1,-\mathrm{Id})\rangle$ and one easily sees that the action of $\left(\mathrm{S}^{1} \times \mathrm{Sp}(3)\right) / \Gamma$ is free if and only if

$$
\text { all } q_{i} \text { 's are odd and } \operatorname{gcd}\left(q_{\tau(1)}+q_{\tau(2)}, q_{\tau(3)}+q_{\tau(4)}\right)=2,
$$

for all permutations $\tau \in S_{6}$. Notice that under a reordering of the integers $q_{i}$, or replacing $q$ with $-q$, one obtains the same manifold.

The manifolds $\mathcal{B}_{q}$ can be written in an alternative way. Since the symmetric space $\mathrm{SU}(6) / \mathrm{Sp}(3)$ can be described as the set of special orthogonal quaternionic structures on $\mathbb{R}^{12}$, one easily sees that the subgroup $\mathrm{SU}(5) \subset \mathrm{SU}(6)$ acts transitively with isotropy group $\mathrm{Sp}(2)$ (see e.g. [Ke]). Indeed, this follows from the usual normal form for such structures since one can fix the first vector arbitrarily. If $\mathrm{SU}(5)$ is the subgroup that fixes $e_{0}=(1,0, \ldots, 0) \in \mathbb{C}^{6}$, it follows that $\mathcal{B}_{q}$ becomes a quotient of $\mathrm{SU}(5)$ under the action of $\mathrm{S}^{1} \times \mathrm{Sp}(2)$ :

$$
\mathcal{B}_{q}=\operatorname{diag}\left(z^{q_{1}}, \ldots, z^{q_{5}}\right) \backslash \mathrm{SU}(5) / \operatorname{diag}\left(z^{-q_{0}}, A\right)^{-1},
$$

where $A \in \mathrm{Sp}(2) \subset \mathrm{SU}(4) \subset \mathrm{SU}(5)$ with the embedding of $\mathrm{Sp}(2)$ and $\mathrm{Sp}(3)$ as described above. In this language it is natural to describe the space by the five integers $\bar{q}=\left(q_{1}, \ldots, q_{5}\right)$. Accordingly, we will refer to $\mathcal{B}_{q}$ in either way, namely, by $q \in \mathbb{Z}^{6}$ or $\bar{q} \in \mathbb{Z}^{5}$, and no confusion will arise because of the different number of integers involved. But it is important to observe that, for the $\bar{q} \in \mathbb{Z}^{5}$ notation, aside from ordering and sign, replacing one of the integers by the negative of their sum also gives the same space. E.g., the Berger space can be written as $(1,1,1,1,1)$ or as $(-5,1,1,1,1)$.

This action again has an ineffective kernel $\mathbb{Z}_{2}=\langle(-1,-$ Id $)\rangle$. To make this second description into a true biquotient, we use the diffeomorphism $\mathrm{SU}(5) \simeq \mathrm{U}(5) / \operatorname{diag}(w, \mathrm{Id})$ to rewrite it as

$$
\mathcal{B}_{\bar{q}}=\operatorname{diag}\left(z^{q_{1}}, \ldots, z^{q_{5}}\right) \backslash \mathrm{U}(5) / \operatorname{diag}(w, A)^{-1},
$$

with $z, w \in \mathrm{S}^{1}$ and $A \in \mathrm{Sp}(2)$.

In [Ba] one finds the somewhat different description

$$
\operatorname{diag}\left(z^{q_{1}^{\prime}}, \ldots, z^{q_{5}^{\prime}}\right) \backslash \mathrm{U}(5) / \operatorname{diag}(1, w A)^{-1},
$$

and one sees, by writing both as quotients of $\mathrm{SU}(5)$, that they are related by

$$
4 q_{i}^{\prime}=q_{i}+q_{0}, \quad i=1, \ldots, 5 .
$$

Notice that one needs $q_{i} \equiv \epsilon \bmod 4$ for all $i=1, \ldots, 5$, for a fixed $\epsilon= \pm 1$, in order to rewrite a Bazaikin space as defined in (1.3) into one as defined in (1.4). This turns out to be a strong condition for the positively curved Bazaikin spaces (see Section 4). 
There are two natural fibrations associated to a Bazaikin space. First, $\mathcal{B}_{q}$ is, by definition, the base space of a principal circle bundle:

$$
\mathrm{S}^{1} \rightarrow \mathrm{SU}(6) / \mathrm{Sp}(3) \rightarrow \mathcal{B}_{q}
$$

But notice that the $S^{1}$ action is only free modulo the ineffective $\mathbb{Z}_{2}$ kernel. Second, the total space of this fibration has a further natural fibration

$$
\mathbb{S}^{5} \rightarrow \mathrm{SU}(6) / \mathrm{Sp}(3) \rightarrow \mathbb{S}^{9}
$$

To see this fibration, we use the fact that $\mathrm{SU}(6) / \mathrm{Sp}(3)=\mathrm{SU}(5) / \mathrm{Sp}(2)$. One then obtains a fibration from the inclusions $\mathrm{Sp}(2) \subset \mathrm{SU}(4) \subset \mathrm{SU}(5)$ and the fact that $\mathrm{SU}(4) / \mathrm{Sp}(2)=$ $\mathrm{SO}(6) / \mathrm{SO}(5)=\mathbb{S}^{5}$ and $\mathrm{SU}(5) / \mathrm{SU}(4)=\mathbb{S}^{9}$.

In order to obtain metrics with positive curvature, the symmetric metric has to be modified. Each Bazaikin space has six natural biquotient metrics associated to it. First, one can rewrite a space as in 1.1 in six different ways as in 1.3 by choosing different base points $e_{0}$ along the coordinate axes. This corresponds to removing one of the six integers in $q$. For a space as in (1.3) we have the natural left invariant metric on SU(5) obtained from the biinvariant metric by scaling with factor $<1$ in the direction of $U(4) \subset$ $\mathrm{SU}(5)$ embedded in the last four coordinates. Since $S^{1} \times \mathrm{Sp}(2)$ acts by isometries in this modified metric, it induces a submersion metric on $\mathcal{B}_{q}$.

In [Zi] it was shown (see [DE] for a published version) that a necessary and sufficient condition for one of these metrics to have positive sectional curvature is that

$$
\text { there exists } 0 \leq i_{0} \leq 5 \text { such that } q_{i}+q_{j}>0 \text { (or }<0 \text { ) for all } i_{0} \neq i<j \neq i_{0} \text {. }
$$

It is easy to check that at most one of these six metrics has positive curvature. In the original paper $[\mathrm{Ba}]$, there are four conditions ensuring positive sectional curvature for a metric as defined in (1.4). But notice that condition (c) in [Ba p. 1069] implies the other three and that in the translation (1.5) condition (c) corresponds to $q_{i}>0$ for $i=1, \ldots, 5$. Bazaikin's condition is again restrictive. In fact, around $7 \%$ of all examples satisfy both of Bazaikin's extra assumptions; see Section 4.

We point out that the change 1.1 in the description of the Bazaikin spaces turns out to be more than cosmetic. It simplifies the expressions for the invariants and the proof of our theorems significantly. Notice that for the symmetric polynomials one has $\sigma_{i}(q)=$ $\sigma_{i}(\bar{q})-\sigma_{1}(\bar{q}) \sigma_{i-1}(\bar{q})$.

\section{Homotopy invariants}

In this section we discuss the topology of the Bazaikin spaces. We start with the cohomology ring. Although already computed in $[\mathrm{Ba}]$, we present a proof here since it is much simpler and some of the information obtained will be used again in the computation of the Pontryagin classes. 
Proposition 2.1 ([B] $]$ ). The Bazaikin space $\mathcal{B}_{q}$ is simply connected and the non-vanishing cohomology groups are given by

$$
\begin{aligned}
& H^{0}\left(\mathcal{B}_{q}\right)=H^{2}\left(\mathcal{B}_{q}\right)=H^{4}\left(\mathcal{B}_{q}\right)=H^{9}\left(\mathcal{B}_{q}\right)=H^{11}\left(\mathcal{B}_{q}\right)=H^{13}\left(\mathcal{B}_{q}\right)=\mathbb{Z}, \\
& H^{6}\left(\mathcal{B}_{q}\right)=H^{8}\left(\mathcal{B}_{q}\right)=\mathbb{Z}_{s},
\end{aligned}
$$

with $8 s=\left|\sigma_{3}(q)\right|$. The ring structure is determined by the fact that if $u \in H^{2}\left(\mathcal{B}_{q}\right)$ is a generator, then $u^{i}$ is a generator of $H^{2 i}\left(\mathcal{B}_{q}\right)$ for $i=2,3,4$.

Proof. As discussed in Section 1 , let $G=\mathrm{SU}(6), H=\mathrm{S}^{1}$ and $K=\mathrm{Sp}(3)$ with embeddings $f_{1}: H \rightarrow G$ given by $f_{1}(z)=\operatorname{diag}\left(z^{q_{0}}, \ldots, z^{q_{5}}\right) \in \mathrm{SU}(6)$ and $f_{2}: K \rightarrow G$ the canonical embedding of $\mathrm{Sp}(3) \subset \mathrm{SU}(6)$. Furthermore, assume that the biquotient action of $(H \times K) / \Gamma$ on $G$ is free, where $\Gamma=\mathbb{Z}_{2}=\langle(-1,-$ Id $)\rangle$.

For brevity, $L M$, in the following, will always denote the direct product $L \times M$ of the connected Lie groups $L$ and $M$. Furthermore, $B_{L}$ will denote the classifying space of $L$. Choose a contractible space $E$ on which $G G$ acts freely. Hence $H K$ and $H$ or $K$ also act freely with quotient their respective classifying space.

The fibration in 1.6 induces the fibration

$$
G / K \rightarrow \mathcal{B}_{q} \rightarrow B_{H^{\prime}},
$$

where $H^{\prime}=H /\{ \pm 1\}$ is the free action of $S^{1}$ on $G / K$. On the other hand, the Serre spectral sequence of the fibration 1.7 implies that $H^{*}(G / K, \mathbb{Z})=H^{*}\left(\mathbb{S}^{5} \times \mathbb{S}^{9}, \mathbb{Z}\right)$. Hence in the spectral sequence of the fibration 2.2 one has only two non-vanishing differentials:

$$
d_{6}: E_{5}^{0,5}=\mathbb{Z} \rightarrow E_{5}^{6,0}=\mathbb{Z}, \quad d_{10}: E_{11}^{0,9}=\mathbb{Z} \rightarrow E_{11}^{10,0}=\mathbb{Z}_{s} .
$$

The differential $d_{6}$ is multiplication by some integer $s$ and, together with Poincaré duality, it follows that the cohomology ring of $\mathcal{B}_{q}$ is as claimed in the Theorem with $H^{6}\left(\mathcal{B}_{q}\right)=$ $H^{8}\left(\mathcal{B}_{q}\right)=\mathbb{Z}_{s}$. It remains to determine the integer $s$. For this purpose we use the naturality of differentials in the following commutative diagram of fibrations:

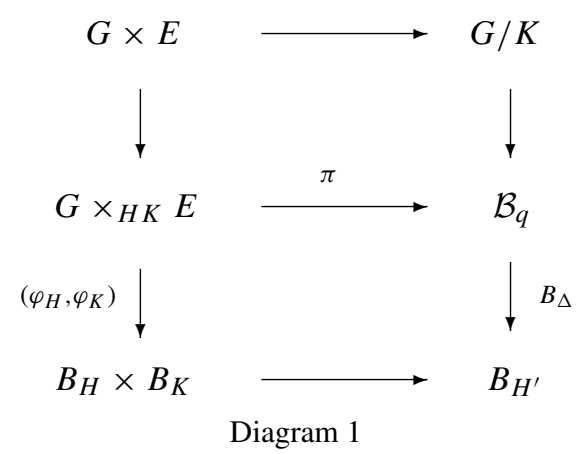

Here $H K$ acts freely on $G \times E$ via a diagonal action consisting of the left and right action on $G$ and the free action on $E$. The projection onto the second factor $G \times{ }_{H K} E \rightarrow$ 
$B_{H} \times B_{K}$ is the classifying map of this $H K$ principal bundle and defines the left hand side fibration. All horizontal maps are the natural projections.

We first claim that the differential $d_{6}^{\prime}$ in the left hand side fibration determines $d_{6}$. To see this, observe that the projection $G \times E \rightarrow G / K$ induces an isomorphism in dimension 5. This follows by using the edge homomorphism in the spectral sequence of $G \times E \rightarrow G / K \rightarrow B_{K}$ since the differentials on $E_{5}^{0,5}=\mathbb{Z}$ vanish for dimension reasons. Furthermore, since $H \rightarrow H^{\prime}$ is given by $z \mapsto z^{2}$, the homomorphism $H^{*}\left(B_{H^{\prime}}\right) \rightarrow$ $H^{*}\left(B_{H}\right)$ is, via transgression, multiplication by 2 in dimension 2 and hence by $2^{i}$ in dimension $2 i$. This describes the induced map $H^{*}\left(B_{H^{\prime}}\right) \rightarrow H^{*}\left(B_{H} \times B_{K}\right)$ and shows that $d_{6}^{\prime}$ determines $d_{6}$.

To compute the differential $d_{6}^{\prime}$, we use naturality with respect to another commutative diagram of fibrations, modifying the method used in [E3] for Eschenburg spaces:

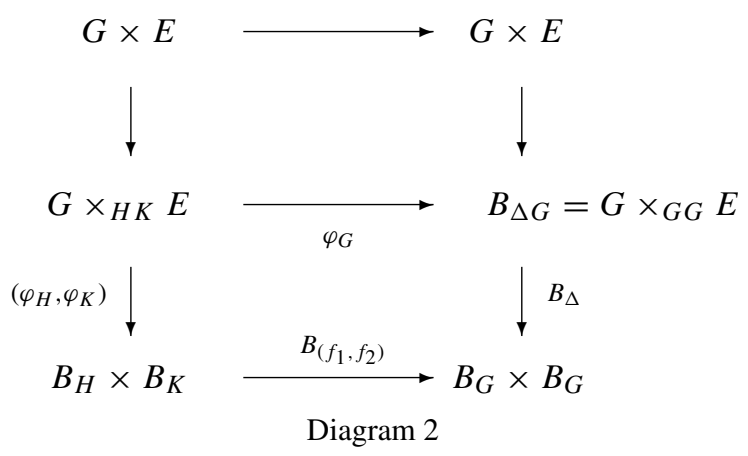

The right hand side fibration comes from the $G G$ principal bundle associated to the free diagonal action of left and right multiplication on $G$ and the free action on $E$. Notice that $G \times{ }_{G G} E=E / G=B_{G}$.

Since all Lie groups involved have no torsion in their cohomology, all remaining computations can be done with integer coefficients, and all cohomology groups are to be understood with $\mathbb{Z}$ coefficients. It is well known that $H^{*}(G)=H^{*}(\mathrm{SU}(6))$ is the exterior algebra $\Lambda\left(y_{2}, \ldots, y_{6}\right)$ and $H^{*}\left(B_{G}\right)$ the polynomial algebra $P\left[\bar{y}_{2}, \ldots, \bar{y}_{6}\right]$. Here, $y_{i}$ has degree $2 i-1$ and its transgression $\bar{y}_{i}$ has degree $2 i$. If $T_{G}$ is the maximal torus of $G$ with coordinates $\left(t_{1}, \ldots, t_{6}\right), \sum t_{i}=0$, we identify $t_{i}$, by abuse of notation, with the elements $t_{i} \in H^{1}\left(T_{G}\right)$ and hence $\bar{t}_{i} \in H^{2}\left(B_{T_{G}}\right)$. We then have $H^{*}\left(B_{T_{G}}\right)=P\left[\bar{t}_{1}, \ldots, \bar{t}_{6}\right]$ and $H^{*}\left(B_{G}\right)=H^{*}\left(B_{T_{G}}\right)^{W_{G}}$, where $W_{G}$ is the Weyl group of $G$. A basis of the algebra of $W_{G}$-invariant elements is given by the elementary symmetric polynomials $\sigma_{i}(\bar{t})=$ $\sigma_{i}\left(\bar{t}_{1}, \ldots, \bar{t}_{6}\right)$ and hence we can choose $\bar{y}_{i}=\sigma_{i}(\bar{t})$.

Similarly, for $T_{K}$ we use the coordinates $\left(s_{1}, s_{2}, s_{3}\right)$ and hence $H^{*}\left(B_{K}\right)=$ $H^{*}\left(B_{T_{K}}\right)^{W_{K}}=P\left[\bar{s}_{1}, \bar{s}_{2}, \bar{s}_{3}\right]^{W_{K}}=P\left[b_{1}, b_{2}, b_{3}\right]$, with $b_{i}=\sigma_{i}\left(\bar{s}_{1}^{2}, \bar{s}_{2}^{2}, \bar{s}_{3}^{2}\right)$. Finally, $H^{*}\left(B_{H}\right)=P[\bar{u}]$, where $u$ is the coordinate of the circle $H$.

Under the embeddings $f_{1}: T_{H} \rightarrow T_{G}$ with $f_{1}(u)=\left(q_{0} u, \ldots, q_{5} u\right)$ we have $f_{1}^{*}\left(t_{i}\right)$ $=q_{i} u$ and hence $B_{f_{1}}^{*}\left(\bar{y}_{i}\right)=B_{f_{1}}^{*}\left(\sigma_{i}(\bar{t})\right)=\sigma_{i}(q) \bar{u}^{i}$. Furthermore, $f_{2}: T_{K} \rightarrow T_{G}$ is given by $f_{2}\left(s_{1}, s_{2}, s_{3}\right)=\left(s_{1},-s_{1}, s_{2},-s_{2}, s_{3},-s_{3}\right)$ and clearly $B_{f_{2}}^{*}\left(\bar{y}_{2 i+1}\right)=0$ since $H^{2 i+1}\left(B_{K}\right)=0$. 
As was shown in [E3], the differentials in the spectral sequence for the fibration $B_{\Delta G} \rightarrow B_{G G}$ are given by

$$
d_{2 i}\left(y_{i}\right)=\bar{y}_{i} \otimes 1-1 \otimes \bar{y}_{i},
$$

where we identify $y_{i} \in H^{*}(G)$ with elements in $E_{2}^{0, *}$ and $H^{*}\left(B_{G} \times B_{G}\right)$ with elements in $E_{2}^{*, 0}$. By naturality, the differentials in the spectral sequence for the left hand side fibration in Diagram 2 are given by $d_{2 i}^{\prime}\left(y_{i}\right)=B_{f_{1}}^{*}\left(\bar{y}_{i}\right)-B_{f_{2}}^{*}\left(\bar{y}_{i}\right)$ and hence $d_{6}^{\prime}\left(y_{3}\right)=$ $\sigma_{3}(q) \bar{u}^{3}$. We conclude that $d_{6}^{\prime}: E_{5}^{0,5}=\mathbb{Z} \rightarrow E_{5}^{6,0}=\mathbb{Z}$ is multiplication by $\sigma_{3}(q)$.

This proves our claim since, as we observed earlier, $H^{*}\left(B_{H^{\prime}}\right) \rightarrow H^{*}\left(B_{H}\right)$ is multiplication by 8 in dimension 6 .

In [CEZ] it was shown that for a given positively curved Eschenburg space, there are only finitely many other positively curved Eschenburg spaces with the same cohomology ring. As claimed in Corollary $\mathrm{C}$, the analogous result for Bazaikin spaces is true as well:

Proof of Corollary $C(a)$. The only variable cohomology group is $H^{6}\left(\mathcal{B}_{q}\right)=H^{8}\left(\mathcal{B}_{q}\right)=$ $\mathbb{Z}_{s}$. We reorder the integers and change the sign of $q$ so that $q_{0} \leq q_{1} \leq \cdots \leq q_{5}$ and $\left|q_{2}\right| \leq q_{3}$. The positive curvature condition is then equivalent to $q_{1}+q_{2}>0$. We can rewrite $s$ as

$$
\begin{aligned}
8 s=-\sigma_{3}(q)= & \left(q_{1}+q_{2}\right)^{2}\left(q_{3}+q_{4}+q_{5}\right)+\left(q_{1}+q_{2}\right)\left(\left(q_{3}+q_{4}+q_{5}\right)^{2}+q_{1} q_{2}\right) \\
& +\left(q_{3}+q_{4}\right)\left(q_{4}+q_{5}\right)\left(q_{3}+q_{5}\right),
\end{aligned}
$$

which clearly implies the desired claim.

A more subtle homotopy invariant is given by the linking form which is defined as follows. Let $X$ be a simply connected manifold whose cohomology ring agrees with the cohomology ring of a Bazaikin space for some integer $s$. Throughout this note, we will call such a space a homological Bazaikin space. Consider the Bockstein homomorphism $\beta: H^{5}\left(X, \mathbb{Z}_{s}\right)=\mathbb{Z}_{s} \rightarrow H^{6}(X, \mathbb{Z})=\mathbb{Z}_{s}$, associated to the short exact sequence $0 \rightarrow \mathbb{Z} \stackrel{\cdot s}{\rightarrow} \mathbb{Z} \rightarrow \mathbb{Z}_{s} \rightarrow 0$. The long exact sequence

$$
\cdots \rightarrow H^{5}(X, \mathbb{Z}) \rightarrow H^{5}\left(X, \mathbb{Z}_{S}\right) \stackrel{\beta}{\longrightarrow} H^{6}(X, \mathbb{Z}) \rightarrow \cdots
$$

implies that $\beta$ is an isomorphism since $H^{5}(X, \mathbb{Z})=0$. The linking form is then given by

$$
L: H^{6}(X, \mathbb{Z}) \times H^{8}(X, \mathbb{Z}) \rightarrow \mathbb{Z}_{s}, \quad L(a, b)=\left(\beta^{-1}(a) \cup b\right)([X]),
$$

for a given choice of orientation class $[X] . L$ is clearly determined up to sign by $l k:=$ $L\left(u^{3}, u^{4}\right) \in \mathbb{Z}_{s}$, where $u$ is a generator of $H^{2}(X, \mathbb{Z})$. We now claim that

Theorem 2.4. The linking form of $\mathcal{B}_{q}$ is given by

$$
l k= \pm 32 \sigma_{5}^{-1} \in \mathbb{Z}_{s},
$$

with $\sigma_{5}$ and $s$ as in Theorem B. 
Proof. The number $l k$ can be described in another fashion. Let $X$ be a homological Bazaikin space. Define an $S^{1}$ principal bundle $P$ over $X$ by requiring that its Euler class $e(P)$ is a generator of $H^{2}(X)=\mathbb{Z}$. A change in sign for $e(P)$ corresponds to a change of orientation of the circle bundle. Hence $P$ is well defined up to orientation and is determined by the homotopy type of $X$. From the Gysin sequence for $\mathrm{S}^{1} \rightarrow P \rightarrow X$ and Poincaré duality for $P$ it follows that $H^{*}(P, \mathbb{Z})=H^{*}\left(\mathbb{S}^{5} \times \mathbb{S}^{9}, \mathbb{Z}\right)$. We can now consider the spectral sequence of the bundle

$$
P \rightarrow X \rightarrow B_{\mathrm{S}^{1}}
$$

whose isomorphism type is a homotopy invariant of $X$. As in the proof of Proposition 2.1. it is determined by only two differentials which can be non-zero, namely,

$$
d_{6}: E_{5}^{0,5}=\mathbb{Z} \rightarrow E_{5}^{6,0}=\mathbb{Z}, \quad d_{10}: E_{11}^{0,9}=\mathbb{Z} \rightarrow E_{11}^{10,0}=\mathbb{Z}_{s} .
$$

Here $d_{6}$ must be multiplication by $s$ since $H^{6}(X)=\mathbb{Z}_{s}$, and $d_{10}$ must be multiplication by some $v$ with $\operatorname{gcd}(s, v)=1$ since $H^{10}(X)=0$. This homotopy invariant $v$ is related to the linking form. In [Si] one finds a discussion of the corresponding question for the Eschenburg spaces where $H^{*}(P, \mathbb{Z})=H^{*}\left(\mathbb{S}^{3} \times \mathbb{S}^{5}, \mathbb{Z}\right)$ and the non-zero differentials are $d_{3}$ and $d_{5}$. It is shown there that $l k= \pm v^{-1}$. One easily sees that the same proof carries over in our situation. It thus remains to compute the invariant $v$.

In the case of $X=\mathcal{B}_{q}$ the total space $P$ of the circle bundle can be chosen to be $P=$ $\mathrm{SU}(6) / \mathrm{Sp}(3)$. Indeed, by the long homotopy sequence of $\mathrm{Sp}(3) \rightarrow \mathrm{SU}(6) \rightarrow P$ it follows that $\pi_{1}(P)=\pi_{2}(P)=0$ and hence $H^{2}(P)=0$. The Gysin sequence of the circle bundle then implies that the Euler class is a generator of $H^{2}\left(\mathcal{B}_{q}\right)$. The fibration $P \rightarrow X \rightarrow B_{\mathrm{S}^{1}}$ now agrees with the one in 2.2). We can hence compute the differentials in the spectral sequence of this fibration using again Diagrams 1 and 2. As in Proposition 2.1. one shows that $d_{10}$ is determined by $d_{10}^{\prime}$ and that $H^{10}\left(B_{H^{\prime}}\right) \rightarrow H^{10}\left(B_{H}\right)$ is multiplication by 32 . Furthermore, one has $B_{f_{1}}^{*}\left(\bar{y}_{5}\right)=\sigma_{5}(q) \bar{u}^{5}$ and $B_{f_{2}}^{*}\left(\bar{y}_{5}\right)=0$ and hence $d_{10}^{\prime}\left(y_{5}\right)=\sigma_{5}(q) \bar{u}^{5}$. As in Proposition 2.1, this proves our claim.

There is another homotopy invariant associated to a homological Bazaikin space. To describe it, consider, as in the proof of Theorem 2.4 the total space $P$ of the circle bundle with Euler class a generator in $H^{2}(X)$. As explained there, $P$ is simply connected with $H^{*}(P, \mathbb{Z})=H^{*}\left(\mathbb{S}^{5} \times \mathbb{S}^{9}, \mathbb{Z}\right)$. Hence one can describe $P$ as a $C W$-complex

$$
P=\mathbb{S}^{5} \cup e^{9} \cup \cdots,
$$

with cells in dimension $5,9, \ldots$ The attaching map $\partial\left(e^{9}\right)=\mathbb{S}^{8} \rightarrow \mathbb{S}^{5}$ is an element $\alpha \in \pi_{8}\left(\mathbb{S}^{5}\right)=\mathbb{Z}_{24}$ (cf. [Ha, To]) and hence $\pi_{8}(P)=\mathbb{Z}_{28} /\langle\alpha\rangle$. Since $\pi_{8}(X)=\pi_{8}(P)$, the group $\pi_{8}(X)$ must be a quotient of $\mathbb{Z}_{24}$ as well. Thus, homological Bazaikin spaces naturally fall into eight different homotopy types.

For the Bazaikin spaces, we claim that they belong to the class where $\pi_{8}(X)=0$. Indeed, the long homotopy sequence for $\mathrm{Sp}(3) \rightarrow \mathrm{SU}(6) \rightarrow P$ :

$$
\cdots \rightarrow \pi_{8}(\mathrm{SU}(6)) \rightarrow \pi_{8}(P) \rightarrow \pi_{7}(\mathrm{Sp}(3)) \rightarrow \pi_{7}(\mathrm{SU}(6)) \rightarrow \cdots,
$$


together with the fact that $\pi_{7}(\mathrm{SU}(6))=\pi_{7}(\mathrm{Sp}(3))=\mathbb{Z}$ and $\pi_{8}(\mathrm{SU}(6))=0$ ([Mi]), implies that $\pi_{8}(P)=0$ or $\mathbb{Z}$ and thus vanishes.

There is a second natural family of homological Bazaikin spaces. They depend on integers $a=\left(a_{1}, \ldots, a_{3}\right)$ and $b=\left(b_{1}, \ldots, b_{5}\right)$, and are described as a quotient

$$
M_{a, b}=\mathbb{S}^{5} \times \mathbb{S}^{9} / \mathrm{S}_{a, b}^{1}
$$

under the circle action

$$
\begin{aligned}
\left(\left(z_{1}, z_{2}, z_{3}\right),\left(w_{1}, \ldots, w_{5}\right)\right) & \in \mathbb{S}^{5} \times \mathbb{S}^{9} \\
& \rightarrow\left(\left(e^{i a_{1} \theta} z_{1}, \ldots, e^{i a_{3} \theta} z_{3}\right),\left(e^{i b_{1} \theta} w_{1}, \ldots, e^{i b_{5} \theta} w_{5}\right)\right) .
\end{aligned}
$$

From the fibration $\mathrm{S}^{1} \rightarrow \mathbb{S}^{5} \times \mathbb{S}^{9} \rightarrow M_{a, b}$ it follows that $\pi_{8}\left(M_{a, b}\right)=\mathbb{Z}_{24}$. Summarizing, we have

Proposition 2.6. The Bazaikin spaces belong to the homotopy type $\pi_{8}(X)=0$ and the manifolds $M_{a, b}$ to the homotopy type $\pi_{8}(X)=\mathbb{Z}_{24}$.

In the case where $a_{1}=a_{2}=a_{3}$ and $b_{1}=\cdots=b_{5}$ the manifold $M_{a, b}$ is homogeneous under the transitive action of $\mathrm{SU}(3) \times \mathrm{SU}(5)$ on $\mathbb{S}^{5} \times \mathbb{S}^{9}$ since it commutes with the circle action.

Remark 2.7. By combining the long homotopy sequences of the above fibrations, one easily shows that the homotopy groups $\pi_{i}=\pi_{i}\left(\mathcal{B}_{q}\right), i \leq 13$, are given by

$$
\begin{aligned}
& \pi_{i}=0 \quad \text { for } i=1,3,4,8,10,11, \\
& \pi_{6}=\pi_{7}=\pi_{13}=\mathbb{Z}_{2}, \quad \pi_{12}=\mathbb{Z}_{360} \quad \text { and } \quad \pi_{2}=\pi_{5}=\pi_{9}=\mathbb{Z}
\end{aligned}
$$

(cf. [To]). In particular, one sees that the Bazaikin spaces cannot be distinguished from each other by their low dimensional homotopy groups.

As we will see in the next section, there is another homotopy invariant given by $p_{1}$ mod 24 which divides the Bazaikin spaces into two different homotopy types.

\section{Diffeomorphism invariants}

The next natural set of invariants are the Pontryagin classes, which depend on the diffeomorphism type. We now show:

Theorem 3.1. The Pontryagin classes $p_{1}$ and $p_{2}$ of $\mathcal{B}_{q}$ are given by

$$
p_{1}=-\sigma_{2} \in H^{4}\left(\mathcal{B}_{q}\right)=\mathbb{Z}, \quad 8 p_{2}=3 \sigma_{2}^{2}-\sigma_{4} \in H^{8}\left(\mathcal{B}_{q}\right)=\mathbb{Z}_{s},
$$

with $\sigma_{i}$ and $s$ as in Theorem $\mathrm{B}$. 
Proof. To compute the Pontryagin classes, we modify [Si, Theorem 4.2] to the situation in the proof of Proposition 2.1 As described there, we have a projection $\pi: X=G \times_{H K} E$ $\rightarrow \mathcal{B}_{q}$ and an $H K$ principal bundle with classifying map $\left(\varphi_{H}, \varphi_{K}\right): X \rightarrow B_{H} \times B_{K}$. In [Si] the following vector bundles were introduced: $\alpha_{H}=(G / K) \times_{H} \mathfrak{h}$ where $H$ acts on $G / K$ on the left and on $\mathfrak{h}$ via the adjoint representation, and similarly $\alpha_{K}=(H \backslash G) \times_{K} \mathfrak{k}$; and $\alpha_{G}=(H \backslash G) \times(G / K) \times_{G} \mathfrak{g}$ where $G$ acts on $(H \backslash G) \times(G / K)$ via $g \cdot\left(H g_{1}, g_{2} K\right)=$ $\left(H g_{1} g^{-1}, g g_{2} K\right)$ and on $\mathfrak{g}$ via the adjoint representation. The projection onto the first factor shows that they are all vector bundles over $\mathcal{B}_{q}=H \backslash G / K$. Although the action of $H K$ on $G$ is only almost free, one easily sees that the proof of [Si, Theorem 3.2] carries over to show that the tangent bundle $\tau$ of $\mathcal{B}_{q}$ satisfies

$$
\tau \oplus \alpha_{H} \oplus \alpha_{K}=\alpha_{G}
$$

Since the action is only almost free, [Si] Theorem 4.2] cannot be applied directly, but can be modified as follows. Define $\bar{\alpha}_{H}=\left(G \times{ }_{K} E\right) \times_{H} \mathfrak{h}$ with $H$ acting on $G$ on the left and similarly $\bar{\alpha}_{K}=\left(G \times_{H} E\right) \times_{K} \mathfrak{k}$ and $\bar{\alpha}_{G}=\left[\left(G \times_{H} E\right) \times\left(G \times_{K} E\right)\right] \times_{G} \mathfrak{g}$ as vector bundles over $X$. Clearly $\pi^{*}\left(\bar{\alpha}_{H}\right)=\alpha_{H}$ and similarly for the others so that we obtain

$$
\pi^{*}(\tau) \oplus \bar{\alpha}_{H} \oplus \bar{\alpha}_{K}=\bar{\alpha}_{G}
$$

We can now use the usual formula for the Pontryagin classes of a homogeneous vector bundle $\alpha_{L}=P \times{ }_{L} V$ associated to the $L$ principal bundle $P \rightarrow P / L=B$ :

$$
p\left(\alpha_{L}\right)=1+p_{1}+p_{2}+\cdots=\varphi_{L}^{*}(a), \quad a=\prod\left(1+\alpha_{i}^{2}\right),
$$

where $\alpha_{i}$ runs through the positive weights of the representation of $L$ on $V$, and $\varphi_{L}$ : $B \rightarrow B_{L}$ is the classifying map of the $L$ principal bundle. Here we have identified $\alpha_{i} \in$ $H^{1}\left(T_{L}\right) \cong H^{2}\left(B_{T_{L}}\right)$ and hence $a \in H^{*}\left(B_{T_{L}}\right)^{W_{L}} \cong H^{*}\left(B_{L}\right)$.

The vector bundles $\bar{\alpha}_{H}, \bar{\alpha}_{K}, \bar{\alpha}_{G}$ are associated to a principal bundle and the weights are the roots of the corresponding Lie group. Thus $p\left(\bar{\alpha}_{H}\right)=1$, and we obtain

$$
p\left(\pi^{*}(\tau)\right)=\varphi_{G}^{*}(a) \varphi_{K}^{*}\left(b^{-1}\right), \quad a=\prod_{\alpha_{i} \in \Delta_{G}^{+}}\left(1+\alpha_{i}^{2}\right), \quad b=\prod_{\beta_{i} \in \Delta_{K}^{+}}\left(1+\beta_{i}^{2}\right) .
$$

The positive roots $\Delta_{G}^{+}$for $G=\mathrm{SU}(6)$ are $t_{i}-t_{j}, 1 \leq i<j \leq 6$, with $\sum t_{i}=0$, and a computation shows that

$$
a=1-12 \bar{y}_{2}+60 \bar{y}_{2}^{2}+12 \bar{y}_{4}+\cdots,
$$

where $\bar{y}_{i}=\sigma_{i}\left(\bar{t}_{1}, \ldots, \bar{t}_{6}\right)$ are the elementary symmetric polynomials in $\bar{t}_{i} \in H^{2}\left(B_{T_{G}}\right)$. Similarly, the positive roots for $K=\mathrm{Sp}(3)$ are $s_{i} \pm s_{j}, 2 s_{i}$ with $1 \leq i<j \leq 3$ and hence

$$
b=1+8 b_{1}+22 b_{1}^{2}+14 b_{2}+\cdots \quad \text { and } \quad b^{-1}=1-8 b_{1}+42 b_{1}^{2}-14 b_{2}+\cdots,
$$

with $b_{i} \in H^{2}\left(B_{T_{K_{1}}}\right)$ defined as in the proof of Proposition 2.1 . 
From the spectral sequence computation in Proposition 2.1 it follows that $\varphi_{G}^{*}\left(\bar{y}_{i}\right)=$ $B_{f_{1}}^{*}\left(\bar{y}_{i}\right)=\sigma_{i}(q) \bar{u}^{i}$. Furthermore, one shows that $B_{f_{2}}^{*}\left(\bar{y}_{2}\right)=-b_{1}$ and $B_{f_{2}}^{*}\left(\bar{y}_{4}\right)=b_{2}$ and hence $\varphi_{K}^{*}\left(b_{1}\right)=-\sigma_{2} \bar{u}^{2}$ and $\varphi_{K}^{*}\left(b_{2}\right)=\sigma_{4} \bar{u}^{2}$. Altogether,

$$
\begin{aligned}
\varphi_{G}^{*}(a) \varphi_{K}^{*}\left(b^{-1}\right) & =\left(1-12 \sigma_{2} \bar{u}^{2}+12\left(5 \sigma_{2}^{2}+\sigma_{4}\right) \bar{u}^{4}+\cdots\right)\left(1+8 \sigma_{2} \bar{u}^{2}+14\left(3 \sigma_{2}^{2}-\sigma_{4}\right) \bar{u}^{4}+\cdots\right) \\
& =1-4 \sigma_{2} \bar{u}^{2}+2\left(3 \sigma_{2}^{2}-\sigma_{4}\right) \bar{u}^{4}+\cdots,
\end{aligned}
$$

which computes the Pontryagin class for $\pi^{*}(\tau)$. Since $\pi^{*}(p(\tau))=p\left(\pi^{*}(\tau)\right)$, the Pontryagin classes for $\tau$ will now be determined by the homomorphisms $\pi^{*}: H^{i}(\mathcal{B}) \rightarrow$ $H^{i}(X)$, where $i=4,8$. Recall that in the proof of Proposition 2.1 we showed that $\pi^{*}: H^{2}\left(B_{H^{\prime}}\right) \rightarrow H^{2}\left(B_{H}\right)$ is multiplication by 2 in dimension 2 . On the other hand, using Diagram 2 we showed that $H^{2}(X)$ is generated by $\bar{y} \in H^{2}\left(B_{H}\right)$, whereas Diagram 1 implies that $H^{2}\left(\mathcal{B}_{q}\right)=H^{2}\left(B_{H^{\prime}}\right)$. Hence $\pi^{*}$ is multiplication by 4 in dimension 4 , and by 16 in dimension 8 . Combining all of these proves our claim.

Notice that the first Pontryagin class, interpreted as an integer, has a well defined sign since $u^{2}$ is a uniquely defined generator in $H^{4}\left(\mathcal{B}_{q}\right)=\mathbb{Z}$. Since the rational Pontryagin classes are not only diffeomorphism invariants, but also homeomorphism invariants, so is the integer $p_{1}=-\sigma_{2}(q)$. Furthermore, a theorem of Hirzebruch ([i]i]) says that $p_{1}$ mod 24 is a homotopy invariant. For this, we have the following.

Corollary 3.2. For any Bazaikin space $\mathcal{B}_{q}$, the following holds:

(a) The first Pontryagin class satisfies $p_{1}=7$ or $15 \bmod 24$. Moreover, up to order and sign, we have:

(a.1) $p_{1}=15 \bmod 24$ if and only if $q \bmod 3=(1,1,1,1,1,1)$ or $(1,1,1,0,0,0)$;

(a.2) $p_{1}=7 \bmod 24$ if and only if $q \bmod 3=(1,1,1,1,-1,0)$;

(b) The order $s$ of $H^{6}\left(\mathcal{B}_{q}\right)=\mathbb{Z}_{s}$ satisfies $s= \pm 1 \bmod 6$.

Proof. Since all $q_{i}$ 's are odd, by $(1.2)$ we have $q \bmod 4= \pm(1,1,1,1,1,-1)$. Then we easily see that $p_{1}=7 \bmod 8$ and that $s$ is odd. Again by $(1.2)$, the only possibilities for $q$ mod 3 are $(1,1,1,1,1,1),(1,1,1,0,0,0)$ or $(1,1,1,1,-1,0)$ up to ordering and sign of $q$. In all cases we obtain $s= \pm 1 \bmod 3$. An easy computation verifies the remaining claims.

Thus Bazaikin spaces naturally fall into two homotopy types. It is also known that $p_{2}+2 p_{1}^{2}$ is a homotopy invariant $\bmod 5([\overline{\mathrm{Wu}}])$. But this does not give any further information. Indeed, using (1.2) we see that $s \bmod 5=0$ if and only if $q \bmod 5= \pm(\epsilon, \epsilon, \epsilon, \epsilon, \epsilon, 0)$, for $\epsilon=1,2$. This easily gives $p_{1} \bmod 5=p_{2} \bmod 5=0$.

With the expression for the above topological invariants, we are in a position to give the

Proof of Theorem $A$. Let $M=G / H$ be a homogeneous space which we assume is homotopy equivalent to $\mathcal{B}_{q}$ for some $q$. Since it is simply connected, it is well known that there exists a semisimple subgroup of $G$ which also acts transitively. We can hence assume that $G$ is semisimple. We can furthermore assume that $G$ is simply connected by 
making the action on $G / H$ ineffective if necessary. Since $M$ is simply connected, this implies that $H$ is connected. From the long homotopy sequence of the fibrations (1.6) and (1.7), together with the fact that the rational homotopy groups of an odd $n$-dimensional sphere are only non-zero in dimension $n$, it follows that $\pi_{i}\left(\mathcal{B}_{q}\right) \otimes \mathbb{Q}=0$ for $i \neq 2,5,9$ and that it is equal to $\mathbb{Q}$ for $i=2,5,9$. Hence the same holds for $G / H$. Now recall that for every compact Lie group $L$ one has $H^{*}(L, \mathbb{Q})=H^{*}\left(S^{n_{1}} \times \cdots \times S^{n_{k}}, \mathbb{Q}\right)$ where $L$ has rank $k$ and the integers $n_{i}$ are called the exponents of $L$. These exponents are well known for each compact simple Lie group (cf. [KZ, Table 1.4] for a complete list). In particular, they are all odd, 1 occurs with multiplicity $r$ if and only if the center of $L$ is $r$ dimensional, and 3 occurs with multiplicity $m$ if and only if $L$ has $m$ simple factors. Thus, from the long exact homotopy sequence for $H \rightarrow G \rightarrow M$ tensored with $\mathbb{Q}$, and the fact that $G$ contains no $\mathrm{S}^{1}$ factors, it follows that $H=H^{\prime} \cdot \mathrm{S}^{1}$ with $H^{\prime}$ semisimple, and that $G$ and $H^{\prime}$ have the same number of simple factors. Furthermore, there must be an extra $\mathbb{S}^{5}$ and $\mathbb{S}^{9}$ in the sphere decomposition of $G$ compared to the one for $H^{\prime}$. We can now use [KZ, Lemma 1.4], which states that a homomorphism of Lie groups $f: A \rightarrow B$ such that $f_{*}: \pi_{*}(A) \otimes \mathbb{Q} \rightarrow \pi_{*}(B) \otimes \mathbb{Q}$ is onto must itself be onto as a Lie group homomorphism as well. Hence, if $G$ has at least three simple factors, $G=G_{1} \times G_{2} \times G_{3} \times \cdots$, and the extra 5- and 9-spheres are contained in $G_{1} \times G_{2}$, the projection of $H$ onto $G_{3} \times \cdots$ must be onto, which implies that $G_{1} \times G_{2}$ already acts transitively on $G / H$. Hence we can assume that $G$ and $H^{\prime}$ both have one or two simple factors.

We now look at the list of exponents for simple Lie groups, and use the usual abbreviations $A_{n}=\mathrm{SU}(n+1), C_{n}=\mathrm{Sp}(n), D_{n}=\mathrm{SO}(2 n)$. By abuse of notation $C_{n}$ will also stand for $B_{n}=\mathrm{SO}(2 n+1)$ since they have the same exponents. Due to the low dimensional isomorphisms $D_{3}=A_{3}$ and $C_{1}=A_{1}=D_{1}$, we can assume that $n \geq 1$ for $C_{n}$, $n \geq 2$ for $A_{n}$, and $n \geq 4$ for $D_{n}$. Now observe that the only simple Lie groups which contain a 5 -sphere are $A_{n}$ for some $n \geq 2$, and the only ones which contain a 9-sphere are $A_{n}, n \geq 4$, or $D_{5}$. One easily obtains the following list of pairs $\left(G, H^{\prime}\right)$ which have all of the above properties:

(I) $\left(G, H^{\prime}\right)$ is one of (a) $\left(A_{4}, C_{2}\right)$ or (b) $\left(A_{5}, C_{3}\right)$;

(II) $\left(G, H^{\prime}\right)=\left(G_{1} G_{2}, H_{1} H_{2}\right)$ is one of the following:

(a) $\left(A_{3} D_{5}, C_{2} C_{4}\right)$, (b) $\left(A_{2} D_{5}, C_{1} C_{4}\right)$, (c) $\left(A_{3} A_{4}, C_{2} A_{3}\right)$, or (d) $\left(A_{2} A_{4}, C_{1} A_{3}\right)$;

(III) $\left(G, H^{\prime}\right)=\left(G_{1} L, L H_{1}\right)$, where $\left(G_{1}, H_{1}\right)$ is one of the cases in (I).

Using the possible embeddings of classical Lie groups into each other, as well as the low dimensional isomorphisms and the replacement of $C_{n}$ by $B_{n}$ discussed above, we see that none of the simple factors of $H^{\prime}$ can be embedded diagonally in $G_{1} G_{2}$. Hence, in case of (II) and (III), $G / H^{\prime}$ is automatically a product homogeneous space $\left(G_{1} / H_{1}\right) \times$ $\left(G_{2} / H_{2}\right)$ with the order indicated in the list. Since we also have a circle inside $H$, we need the further property that the normalizer of $H_{1}$ in $G_{1}$ or the normalizer of $H_{2}$ in $G_{2}$, or both, are not finite. This excludes (Ib) and (IIa). Case (Ia) uniquely determines the Berger space since $\mathrm{SO}(5) \subset \mathrm{SU}(5)$ has a finite normalizer, and the normalizer of $\mathrm{Sp}(2) \subset \mathrm{SU}(5)$ is one-dimensional. This also excludes case (III), since one needs $H_{1} \subset L \subset G_{1}$ in this case, which is only possible when $L$ contains a circle, contradicting our assumption that $G$ is semisimple. 
For the remaining three cases in (II), we are left with the following possibilities:

$$
\begin{aligned}
& G / H=\left(\mathrm{SU}(3) / \mathrm{SU}(2) \mathrm{S}^{1}\right) \times(\mathrm{SO}(10) / \mathrm{SO}(9))=\mathbb{C P}^{2} \times \mathbb{S}^{9}, \\
& G / H=(\mathrm{SO}(6) / \mathrm{SO}(5)) \times\left(\mathrm{SU}(5) / \mathrm{SU}(4) \mathrm{S}^{1}\right)=\mathbb{S}^{5} \times \mathbb{C P}^{4}, \\
& G / H^{\prime}=(\mathrm{SU}(3) / \mathrm{SU}(2)) \times(\mathrm{SU}(5) / \mathrm{SU}(4))=\mathbb{S}^{5} \times \mathbb{S}^{9}, \quad \text { or } \\
& G / H=(\mathrm{SU}(3) / \mathrm{SO}(3)) \times\left(\mathrm{SU}(5) / \mathrm{SU}(4) \mathrm{S}^{1}\right)=(\mathrm{SU}(3) / \mathrm{SO}(3)) \times \mathbb{C P}^{4} .
\end{aligned}
$$

The two cases where the manifold contains a $\mathbb{C P}^{4}$ factor contradict the rational cohomology ring structure of the Bazaikin space. In (IId) the circle in $H$ can be embedded diagonally at slope $(a, b)$ into the two-dimensional normalizer of $H^{\prime}$ in $G$ and we can assume that $\operatorname{gcd}(a, b)=1$. Case IIb can now be interpreted as the special subcase $(a, b)=(1,0)$. Notice also that $a=0$ again contradicts the rational cohomology ring of $\mathcal{B}_{q}$ and we can hence assume $a \geq 1$.

Altogether, we are left with the Berger space and the manifolds $G / H=\mathbb{S}^{5} \times \mathbb{S}^{9} / \mathrm{S}_{a, b}^{1}$, where the circle $S_{a, b}^{1}$ acts on $\mathbb{S}^{5} \times \mathbb{S}^{9}$ as a Hopf action on each sphere, but with relative speed $a$ on the first factor and $b$ on the second factor. These are special cases of the manifolds $M_{a, b}$ discussed in Proposition 2.6 where we showed that the Bazaikin spaces and the manifolds $\mathbb{S}^{5} \times \mathbb{S}^{9} / \mathrm{S}_{a, b}^{1}$ belong to different homotopy types.

So far, we have shown that if a Bazaikin space has the homotopy type of a homogeneous space, the homogeneous space can only be the Berger space. We now need to show that the only Bazaikin space with this property is the Berger space itself.

For a positively curved Bazaikin space, the equation (2.3) easily implies that among the positively curved manifolds $\mathcal{B}_{q}$ the value $s=5$ is only obtained for the Berger space $\bar{q}=(1,1,1,1,1)$. This proves the first part.

For a general Bazaikin space $\mathcal{B}_{q}$, we use the homeomorphism invariant $p_{1}=\frac{1}{2}\|q\|^{2}$. For the Berger space we have $p_{1}=15$, which means that if $\mathcal{B}_{q}$ is homeomorphic to it, then $\max _{i}\left(\left|q_{i}\right|\right) \leq 5$. One now easily sees that $p_{1}=15$ and $s=5$ is only possible when $\bar{q}=(1,1,1,1,1)$. This finishes the proof of the second assertion.

Remark. (a) The proof can easily be modified to show that a homogeneous space which is a homological Bazaikin space, must be a Berger space or one of $\mathbb{S}^{5} \times \mathbb{S}^{9} / \mathrm{S}_{a, b}^{1}$. For this one uses the fact that a homological Bazaikin space has the rational homotopy type of $\mathbb{S}^{9} \times \mathbb{C P}^{2}$ (cf. [BK, Lemma 8.2]), and hence Sullivan's rational homotopy theory (cf. $[\mathrm{GM}]$ ) shows that $\pi_{*}(X) \otimes \mathbb{Q}$ is non-zero (and one-dimensional) only in dimensions $2,5,9$.

(b) A similar argument gives a short proof that a homogeneous space which has the rational cohomology of an Eschenburg space must be an Aloff-Wallach space or $\mathbb{S}^{3} \times$ $\mathbb{S}^{5} / \mathrm{S}_{a, b}^{1}$. The fourth homotopy group distinguishes the two classes (cf. [E1]). This was used in [E1] to show that many positively curved Eschenburg spaces cannot be homotopy equivalent to a homogeneous space. On the other hand, there are some that are homotopy equivalent but not homeomorphic to Aloff-Wallach spaces; see [Sh].

(c) In [WZ] it was shown that the homogeneous spaces $\mathbb{S}^{5} \times \mathbb{S}^{9} / \mathrm{S}_{a, b}^{1}$ satisfy $s=a^{3}$. For a Bazaikin space $\mathcal{B}_{q}$ with $s$ a cube, there hence exist infinitely many homogeneous 
spaces with the same cohomology ring. It happens occasionally for a positively curved Bazaikin space $\mathcal{B}_{q}$ that $s$ is a cube. The one with this property and smallest $s$ is given by $\bar{q}=(5,5,5,13,23)$, which has $s=17^{3}$.

(d) We finally observe that a Bazaikin space may have the same homotopy invariants (as above) as the Berger space, e.g. $\bar{q}=(-89,15,21,31,111)$.

\section{Some computer experiments}

We wrote a $\mathrm{C}++$ program in order to compute all positively curved Bazaikin spaces $\mathcal{B}_{q}$ together with the invariants considered in this paper, and with order $s=\left|H^{6}\left(\mathcal{B}_{q}\right)\right| \leq$ 40000000 . There are 472959576 manifolds in a +20 GB file. Among them, we looked for subsets of spaces matching different invariants:

(i) 3210637 pairs, 38130 triplets, 645 quadruples, 12 5-tuples and one 6-tuple match the homeomorphism invariants $s$ and $p_{1}$.

(ii) 3065 pairs match the homotopy invariants $s, l k$ and $p_{1} \bmod 24$. The first matching pair (with smallest $s$ ) is $\bar{q}=(-11,13,45,67,77)$ and $(-43,45,49,61,79)$, with $s=254.941, l k=86294$ and $p_{1} \bmod 24=7$. No triplet matches the three invariants.

(iii) 365 pairs match $s, p_{1}$ and $p_{2}$, but no triplets. The first matching pair is given by $(-13,33,41,105,137)$ and $(-3,5,77,83,141)$, with $s=999437, p_{1}=62271$ and $p_{2}=949280$. It is interesting that all these pairs, but one, match the first four symmetric functions $\sigma_{i}(q), 1 \leq i \leq 4$. The pair that does not match the first four symmetric functions (in fact, none of them) is $(-123,149,197,201,525)$ and $(-19,21,75,437,437)$ with $s=28864757, p_{1}=646383, p_{2}=25993311$.

(iv) No pair matches the homeomorphism invariants $s, l k$ and $p_{1}$. In the search for such a pair, we went up to $s \leq 100000000$ : 2130601485 positively curved Bazaikin manifolds in a $+100 \mathrm{~GB}$ file. Still, no pair matches the homeomorphism invariants.

(v) In the case of arbitrary (not necessarily positively curved) Bazaikin manifolds, we found several pairs that match $s, l k, p_{1}$ and $p_{2}$, especially for small values of $s$, where $l k$ and $p_{2}$ have weak influence. There is even a quadruple matching all four invariants: $(-53,-11,25,33,77),(-53,-23,25,49,69),(-53,-3,5,49,73)$ and $(-53,-39,41,49,61)$, with $s=1, l k=0, p_{1}=7.807$ and $p_{2}=0$.

(vi) About $7 \%$ of the examples satisfy the (only necessary) conditions given in [Ba] for the Eschenburg metric on $\mathcal{B}_{q}$ to have positive curvature.

The source code of the programs can be found at http://w3.impa.br/ luis/bazaikin/.

Acknowledgments. We would like to thank C. Escher and T. Chinburg for helpful discussions. This work was done while the second author was visiting IMPA and he would like to thank the Institute for their hospitality.

The first author was supported by CNPq-Brazil and the second author by the Francis J. Carey Term Chair, by a grant from the National Science Foundation, and by the Clay Institute. 


\section{References}

[AW] Aloff, S., Wallach, N.: An infinite family of 7-manifolds admitting positively curved Riemannian structures. Bull. Amer. Math. Soc. 81, 93-97 (1975) Zbl 0362.53033 MR 0370624

[AMP1] Astey, L., Micha, E., Pastor, G.: Homeomorphism and diffeomorphism types of Eschenburg spaces. Differential Geom. Appl. 7, 41-50 (1997) Zbl 0877.57016 MR 1441917

[AMP2] Astey, L., Micha, E., Pastor, G.: On the homotopy type of Eschenburg spaces with positive sectional curvature. Proc. Amer. Math. Soc. 132, 3725-3729 (2004) Zbl 1064.53017 MR 2084097

[Ba] Bazăkin, Ya. V.: On a certain family of closed 13-dimensional Riemannian manifolds of positive curvature. Sibirsk. Mat. Zh. 37, 1219-1237 (1996) (in Russian); English transl.: Siberian Math. J. 37, 1068-1085 (1996) Zbl 0874.53034 MR 1440379

[BK] Belegradek, I., Kapovitch, V.: Obstructions to nonnegative curvature and rational homotopy theory. J. Amer. Math. Soc. 16, 259-284 (2003) Zbl 1027.53036 MR 1949160

[BB] Bérard Bergery, L.: Les variétés riemanniennes homogènes simplement connexes de dimension impaire à courbure strictement positive. J. Math. Pure Appl. 55, 47-68 (1976) Zbl 0289.53037 MR 0417987

[Be] Berger, M.: Les variétés riemanniennes homogènes normales simplement connexes à courbure strictement positive. Ann. Scuola Norm. Sup. Pisa 15, 179-246 (1961) Zbl 0101.14201 MR 0133083

[CEZ] Chinburg, T., Escher, C., Ziller, W.: Topological properties of Eschenburg spaces and 3Sasakian manifolds. Math. Ann. 339, 3-20 (2007)

[DE] Dearricott, O., Eschenburg, J.: Totally geodesic embeddings of 7-manifolds in positively curved 13-manifolds. Manuscripta Math. 114, 447-456 (2004)

[E1] Eschenburg, J.: New examples of manifolds with strictly positive curvature. Invent. Math. 66, 469-480 (1982) Zbl 0484.53031 MR 0662603

[E2] Eschenburg, J.: Freie isometrische Aktionen auf kompakten Lie-Gruppen mit positiv gekrümmten Orbiträumen. Schriftenr. Math. Inst. Univ. Münster 32 (1984) Zbl 0551.53024 MR 0758252

[E3] Eschenburg, J.: Cohomology of biquotients. Manuscripta Math. 75, 151-166 (1992) Zbl 0769.53029 MR 1160094

[EKS] Eschenburg, J., Kollross, A., Shankar, K.: Free isometric circle actions on compact symmetric spaces. Geom. Dedicata 103, 35-44 (2003) Zbl 1048.53021 MR 2026835

[GM] Griffiths, P., Morgan, F.: Rational Homotopy Theory and Differential Forms. Progr. Math. 16, Birkhäuser, Boston, MA (1981) Zbl 0474.55001 MR 0641551

[Ha] Hatcher, A.: Algebraic Topology. Cambridge Univ. Press. Cambridge (2002) Zbl 1044.55001 MR 1867354

[Hi] Hirzebruch, F.: A Riemann-Roch theorem for differentiable manifolds. Sém. Bourbaki, exp. 177 (1959). Zbl 0129.15406 MR 1603462

[KZ] Kapovitch, V., Ziller, W.: Biquotients with singly generated rational cohomology. Geom. Dedicata 104, 149-160 (2004) Zbl 1063.53055 MR 2043959

[Ke] Kerr, M.: Some new homogeneous Einstein metrics on symmetric spaces. Trans. Amer. Math. Soc. 348, 153-171 (1996) Zbl 0864.53035 MR 1327258

[KS] Kreck, M., Stolz, S.: Some nondiffeomorphic homeomorphic homogeneous 7-manifolds with positive sectional curvature. J. Differential Geom. 33, 465-486 (1991); Correction, 49, 203-204 (1998) Zbl 0733.53025 MR 1094466

[K1] Kruggel, B.: Kreck-Stolz invariants, normal invariants and the homotopy classification of generalized Wallach spaces. Quart. J. Math. Oxford Ser. (2) 49, 469-485 (1998) Zbl 0928.57033 MR 1660041 
[K2] Kruggel, B.: Homeomorphism and diffeomorphism classification of Eschenburg spaces. Quart. J. Math. Oxford Ser. (2) 56, 553-577 (2005) Zbl 1104.53028 MR 2182466

[Mi] Mimura, M.: Homotopy theory of Lie groups. In: Handbook of Algebraic Topology, North-Holland, Amsterdam, 951-991 (1995) Zbl 0867.57035 MR 1361904

[Sh] Shankar, K.: Strong inhomogeneity of Eschenburg spaces. Michigan Math. J. 50, 125-141 (2002) Zbl 1036.53022 MR 1897037

[Si] Singhof, W.: On the topology of double coset manifolds. Math. Ann. 297, 133-146 (1993) Zbl 0793.57019 MR 1238411

[To] Toda, H.: Composition Methods in Homotopy Groups of Spheres. Ann. of Math. Stud. 49, Princeton Univ. Press, Princeton, NJ (1962) Zbl 0101.40703 MR 0143217

[Wa] Wallach, N.: Compact homogeneous Riemannian manifolds with strictly positive curvature. Ann. of Math. 96, 277-295 (1972) Zbl 0261.53033 MR 0307122

[WZ] Wang, M. Y., Ziller, W.: Einstein metrics on principal torus bundles. J. Differential Geom. 31, 215-248 (1990) Zbl 0691.53036 MR 1030671

[Wu Wu, W.-T.: On Pontrjagin classes II. Amer. Math. Soc. Transl. (2) 92, 63-92 (1970) Zbl 0206.25405

[Zi] Ziller, W.: Homogeneous spaces, biquotients, and manifolds with positive curvature. Lecture notes (1998), unpublished 